\title{
Étude de l'utilisation des modèles A.R.I.M.A. pour la prévision à très court terme de l'énergie journalière produite par électricité de France
}

Revue française d'automatique, d'informatique et de recherche opérationnelle. Recherche opérationnelle, tome 13, $\mathrm{n}^{\circ} 1$ (1979), p. 37-54.

$<$ http://www.numdam.org/item?id=RO_1979_13_1_37_0>

(C) AFCET, 1979, tous droits réservés.

L'accès aux archives de la revue « Revue française d'automatique, d'informatique et de recherche opérationnelle. Recherche opérationnelle » implique l'accord avec les conditions générales d'utilisation (http://www.numdam.org/ legal.php). Toute utilisation commerciale ou impression systématique est constitutive d'une infraction pénale. Toute copie ou impression de ce fichier doit contenir la présente mention de copyright.

\section{Numdam}




\title{
ÉTUDE DE L'UTILISATION DES MODËLES A.R.I.M.A. POUR LA PREVISION A TRÉS COURT TERME DE L'ENERGIE JOURNALIËRE PRODUITE PAR ELECTRICITÉ DE FRANCE $\left({ }^{*}\right)$
}

\author{
par Jean Abadie ( ${ }^{1}$ ) et François Meslier ( ${ }^{1}$ )
}

\begin{abstract}
Résumé. - La qualité des prévisions à très court terme est une donnée fondamentale de la bonne tenue d'une entreprise productrice d'électricité, en particulier en raison de capacités limitées de stockage de l'énergie électrique. Toute amélioration amènera à la fois une diminution des coûts pour le producteur et une augmentation de la qualité de service pour le consommateur.

Nous proposons la description des principaux résultats obtenus par une première utilisation élaborée des techniques développées par Box et Jenkins (Auto Regressive Integrated Moving Average Processes ou ARIMA).
\end{abstract}

\section{INTRODUCTION}

Nous proposons ici le résumé de l'analyse d'une série chronologique qui revêt une importance toute particulière pour Électricité de France, aussi bien pour des cycles de décision à très court terme que pour des études de gestion prévisionnelle et de planification. Le travail que nous avons effectué ([1], [2]) s'est situé d'emblée dans la perspective de la mise au point d'un modèle de prévision à très court terme. Nous indiquons les principales étapes du développement du modèle et donnons des résultats relatifs à ses performances dans des conditions réelles de prévision.

Cette série est relative à la demande d'énergie électrique en France. La figure 1 représente la forme typique de la demande, par pas horaire, sur deux semaines (du lundi matin au dimanche soir): cette courbe s'intitule dans le jargon des électriciens une courbe de charge. L'analyse de ce schéma de principe permet de mettre en évidence quelques caractéristiques simples de la demande à E. D. F. :

1) les niveaux et les formes des lundi, mardi, mercredi, jeudi et vendredi sont extrêmement semblables. Cependant, le lundi se distingue en général

(*) Reçu octobre 1977.

( ${ }^{1}$ Électricité de France, Direction des Études et Recherches;

R.A.I.R.O. Recherche opérationnelle/Operations Research, 0399/0842/1979/37/\$ 1.00

(C) Bordas-Dunod. 


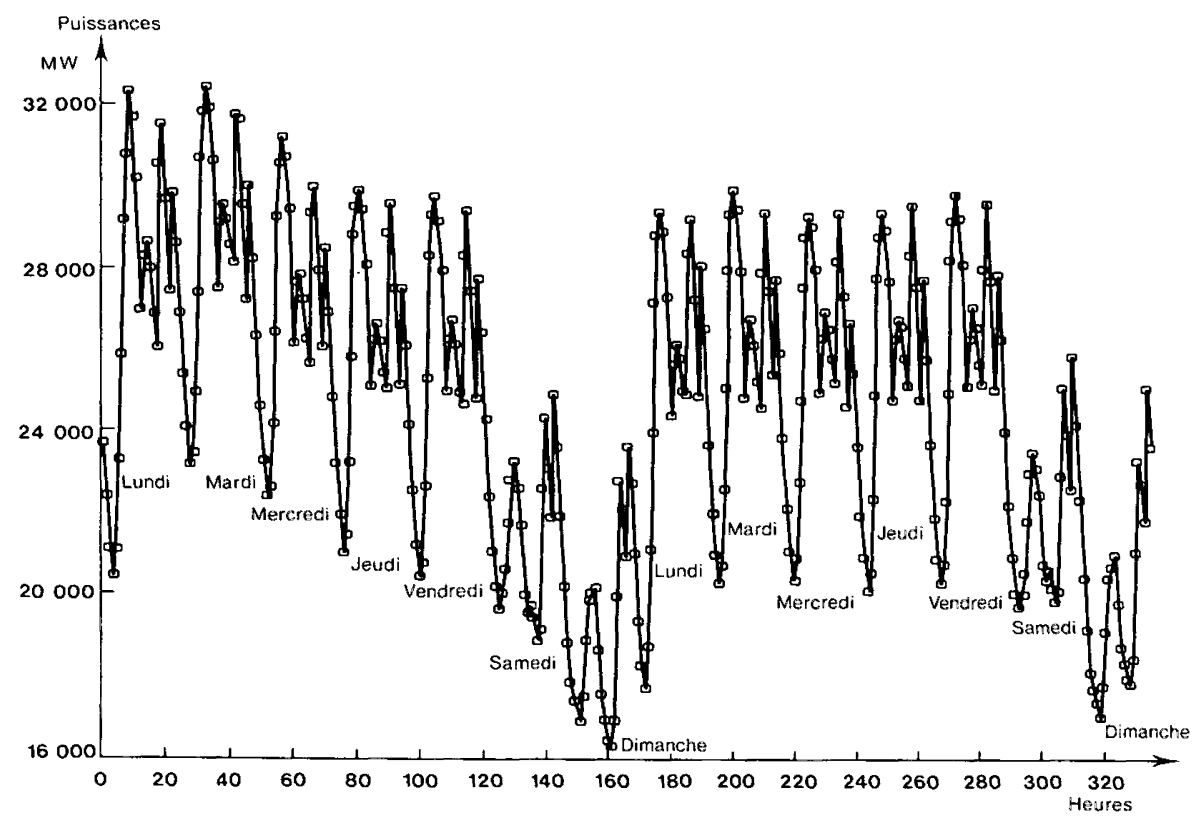

Figure 1. - Exemple de courbes de charges à pas horaire.

par un niveau moyen plus faible que celui des autres " jours ouvrables " (mardi, ..., vendredi);

2) les samedis semblent bien différents, à la fois en forme et en niveau général. Il en est de même pour les dimanches.

Les méthodes actuelles de prévision sont basées sur l'hypothèse de la stabilité de telles caractéristiques au cours du temps. Ceci conduit à effectuer les prévisions à l'aide de divers " facteurs de modulation ». La procédure, résumée sur le tableau 1 (extrait de [3]), comporte deux étapes clefs : prévision de l'énergie journalière à température normale (la température normale d'un jour $t$ se définit comme la moyenne statistique des réalisations des températures de ce jour sur un grand nombre d'années), puis prévision d'une courbe de charge horaire et introduction d'une prévision de température journalière.

Comment peut-on situer notre contribution par rapport aux méthodes actuellement utilisées?

Nous nous sommes limités a priori à l'analyse des seules énergies journalières : la complexité de ce problème apparaîtra très nettement dans le texte. Des travaux complémentaires, sans doute très importants, permettraient d'aborder la prévision de la courbe de charge dans le même esprit. Il est, en 
TABleau 1

Les différents facteurs de modulation

\begin{tabular}{|c|c|c|}
\hline & Étape & Facteur de modulation \\
\hline & $\begin{array}{l}\text { 1. Conversion de la valeur de } \\
\text { l'énergie annuelle en niveaux } \\
\text { d'énergie par jour ouvrable } \\
\text { (moyenne annuelle) }\end{array}$ & $\begin{array}{l}\text { Nombre de jours ouvrables } \\
\text { équivalents }=N\end{array}$ \\
\hline \multirow{3}{*}{ 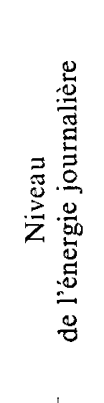 } & $\begin{array}{l}\text { 2. Consommation par jour ouvra- } \\
\text { ble moyen, pour les divers inter- } \\
\text { valles (semaine ou mois) }\end{array}$ & Coefficient saisonnier $=k s$ \\
\hline & $\begin{array}{l}\text { 3. Effet de l'augmentation de la } \\
\text { consommation au cours de la } \\
\text { période annuelle couverte }\end{array}$ & Coefficient de tendance $=r$ \\
\hline & $\begin{array}{l}\text { 4. Conversion de l'énergie du jour } \\
\text { ouvrable moyen d'un intervalle } \\
\text { donné, en énergies pour chaque } \\
\text { type de jour (samedi, diman- } \\
\text { che...) }\end{array}$ & $\begin{array}{l}\text { Facteurs de pondération des } \\
\text { divers types de jours }=p\end{array}$ \\
\hline \multirow{2}{*}{ 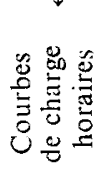 } & $\begin{array}{l}\text { 5. Ventilation de l'énergie journa- } \\
\text { lière en } 24 \text { valeurs de puis- } \\
\text { sances horaires }\end{array}$ & $\begin{array}{l}\text { Courbes de charges rédui- } \\
\text { tes }=\pi_{t}, t=1, \ldots, 24\end{array}$ \\
\hline & $\begin{array}{l}\text { 6. Prise en compte de l'effet clima- } \\
\text { tique }\end{array}$ & $\begin{array}{l}\text { Gradient (variation de la } \\
\text { demande par degré) : }\end{array}$ \\
\hline
\end{tabular}

l'état actuel des résultats obtenus, tout à fait envisageable d'utiliser, à l'aval des modèles obtenus pour les énergies journalières, des coefficients $\pi$ (voir tableau 1) pour définir des courbes de charge prévisionnelles.

Les réalisations des énergies journalières constituent une série chronologique et c'est dans ce seul esprit que nous les avons envisagées.

Les différents types de jours envisagés de manière systématique dans les méthodes actuelles de prévision à court terme sont les lundi, mardi, ..., samedi, dimanche « normaux ». Or il existe de très nombreuses réalisations particulières, correspondant principalement aux jours de fête et aux périodes de vacances et nous en avons tenu compte explicitement dans notre modélisation.

Les modèles envisagés pour expliciter le comportement de la série n'ont pu être ajustés qu'en faisant appel à des méthodes d'optimisation non linéaire assez générales, en raison surtout du nombre très important de réalisations prises en compte et du nombre élevé de paramètres définissant les modèles. L'annexe II donne les idées principales de cet aspect. 
Nous nous sommes limités a priori à la classe des seuls modèles endogènes et ce pour diverses raisons.

Tout d'abord, nous pensons qu'avant de tenter d'expliquer le comportement d'une série par celui d'autres dont elle est réputée dépendre, il importe de retirer au préalable toute la substance que la série apporte en elle-même : autrement dit, la mise au point d'un modèle endogène nous semble de toute manière être la première étape indispensable. La recherche d'éventuels éléments explicatifs extérieurs à la série elle-même ne doit donc porter que sur ce que le modèle endogène ne permet pas de prendre en compte par lui-même.

Ensuite, il est bon de remarquer que, compléter nos modèles endogènes par des modèles exogènes (mise au point de fonctions de transfert) ne constituerait qu'une extension de nos travaux. A cet égard, pour la série étudiée, nous voudrions distinguer deux types de variables exogènes: la prise en compte de l'influence des aléas climatiques améliorerait certainement nos résultats (voir par exemple l'annexe III qui esquisse la détermination d'une fonction de transfert liant énergie journalière et température moyenne journalière). Par contre, nous ne pensons pas que des grandeurs macroéconomiques aient une influence significative pour la prévision à très court terme, l'inertie étant beaucoup plus grande que pour les effets climatiques. Néanmoins, l'évolution du contexte économique peut provoquer des dérives plus ou moins lentes du modèle (modification de la structure de la consommation entre usages industriels et domestiques par exemple); à cet égard, un suivi systématique des qualités prévisionnelles du modèle permet de procéder, si nécessaire, à une réactualisation des paramètres du modèle, voire du modèle lui-même.

Notre propos ne sera pas ici de présenter les principaux éléments théoriques relatifs aux modèles ARIMA (il conviendra par exemple de se reporter à l'ouvrage de Box et Jenkins [4]), mais de montrer sur un exemple en vraie grandeur les résultats qu'on peut obtenir par cette méthode. Nous donnons uniquement, en annexe I, la définition des différentes estimations utilisées dans le texte.

\section{ETUDE DE LA SÉRIE DES ÉNERGIES JOURNALIÈRES}

Nous disposions de statistiques journalières du $1^{\text {er }}$ janvier 1952 au 30 juin 1975. Nous avons ici réservé la dernière année pour réaliser une expérience de simulation de prévisions dans des conditions réelles. Au niveau de l'estimation, nous ne retenons donc que les 8217 premières réalisations, soit du 1.1.1952 au 30.6.1974.

R.A.I.R.O. Recherche opérationnelle/Operations Research 
L'analyse de la série observée et des estimations de ses principales propriétés permet de dégager quelques premières caractéristiques: existence d'une périodicité hebdomadaire, existence d'une tendance, existence d'une périodicité annuelle, amplitude des oscillations de la série qui croît avec le temps, existence d'observations particulières (jours de fête, jours de grève).

REMARQUE : Cette série étant sans doute assez peu connue, nous proposons sur la figure 2 un tracé graphique d'une partie des observations, ce qui permet de préciser quelques-unes des caractéristiques précédentes.

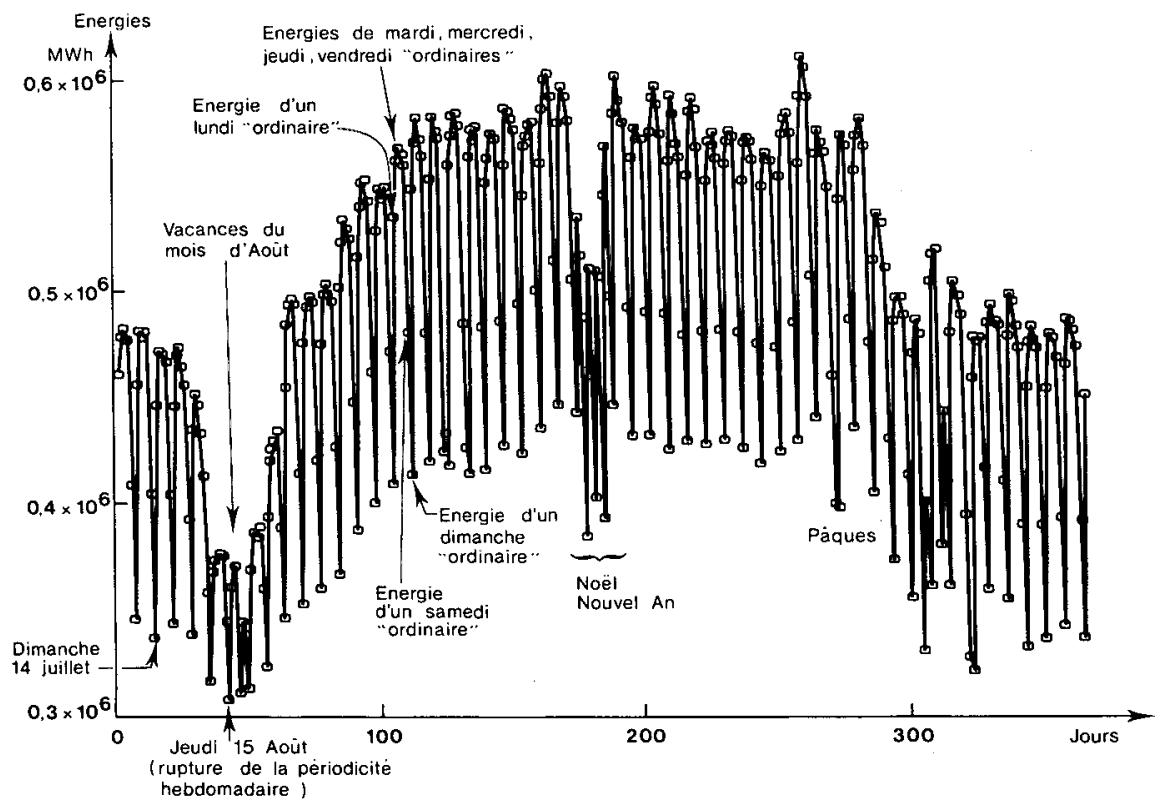

Figure 2. - Extrait de la série des observátions des énergies journalières (du lundi $1^{\text {er }}$ Juillet 1974 au lundi 30 Juin 1975).

\subsection{Recherche d'un ou plusieurs modèles A.R.I.M.A. possibles}

En ce qui concerne l'accroissement de l'amplitude des oscillations de la série, la construction du diagramme de l'étendue en fonction de la moyenne, qui apparaît parfaitement linéaire, conduit à effectuer une transformation logarithmique sur la série initiale (se reporter à [8] pour plus de précision). La série transformée est alors beaucoup plus homogène, avec cependant une légère indication de transformation trop forte.

Pour pouvoir ajuster un modèle ARIMA, il convient ensuite de filtrer les vol. $13, \mathrm{n}^{\circ} 1$, février 1979 
non stationnarités sur la moyenne par des opérateurs différences. Différents essais conduisent à identifier trois modèles possibles (notations en Annexe IV) :

$$
\begin{aligned}
\left(1-\phi_{365} B^{365}\right) \nabla \nabla_{7} \log _{10} x_{t} & =\left(1-\theta_{1} B\right)\left(1-\theta_{7} B^{7}\right) a_{t} \\
\left(1-\phi_{1} B\right) \nabla_{7} \nabla_{365} \log _{10} x_{t} & =\left(1-\theta_{7} B^{7}\right)\left(1-\theta_{365} B^{365}\right) a_{t} \\
\nabla & \nabla_{7} \nabla_{365} \log _{10} x_{t}=\left(1-\theta_{1} B\right)\left(1-\theta_{7} B^{7}\right)\left(1-\theta_{365} B^{365}\right) a_{t}
\end{aligned}
$$

où $a_{t}$ représente pour chacun des modèles le résidu à l'instant $t$.

Comme point de départ, nous avons retenu le modèle (2). Son ajustement par les moindres carrés conduit à :

$$
(1-0,2919 B) \nabla_{7} \nabla_{365} \log _{10} x_{t}=\left(1-0,9686 B^{7}\right)\left(1-0,7068 B^{365}\right) a_{t}
$$

avec :

$$
\sigma\left(a_{t}\right)=0,0263
$$

L'analyse graphique du résidu de ce modèle permet de détecter de très nombreuses réalisations anormales, certaines atteignant jusqu'à 9 écartstypes. Ces résidus particuliers traduisent l'impact des différentes fêtes légales sur la consommation journalière d'énergie électrique.

Il est vraisemblable que le modèle ARIMA est fortement perturbé par ces situations particulières. Leur modélisation permettra d'en comprendre les mécanismes et d'obtenir un modèle ARIMA moins perturbé.

\subsection{Correction des perturbations dues aux différents jours de fête}

Nous ne décrirons pas l'ensemble des essais effectués, car ceci conduirait à analyser un nombre considérable de valeurs numériques. Nous nous contenterons d'indiquer les lignes générales de notre travail de manière à montrer comment le modèle a progressé au cours de cette étape.

L'idée très générale utilisée pour prendre en compte les différentes fêtes est la suivante : par des corrections adéquates, ramener la consommation d'énergie de ces jours particuliers à celle qui aurait été réalisée si le jour avait été normal.

Après plusieurs essais, nous avons retenu une méthode de " correction additive " : soit par exemple $\Gamma$ l'ensemble des indices des jours de fete que nous voulons corriger par un paramètre $\lambda$. Nous estimons simultanément le paramètre $\lambda$ et les paramètres du modèle ARIMA, c'est-à-dire :

$$
\left\{\begin{array}{l}
\underset{\phi_{1}, \theta_{7}, \theta_{365}, i}{\operatorname{MIN}} \sum_{t} a_{t}^{2} \\
\left(1-\phi_{1} B\right) \nabla_{7} \nabla_{365} w_{t}=\left(1-\theta_{7} B^{7}\right)\left(1-\theta_{365} B^{365}\right) a_{t} \\
w_{t}=z_{t} \quad \text { si } t \notin \Gamma \\
w_{t}=z_{t}+\lambda \quad \text { si } \quad t \in \Gamma \\
z_{t}=\log _{10} x_{t}
\end{array}\right.
$$

R.A.I.R.O. Recherche opérationnelle/Operations Research 
Pour certains jours de fête, ce type de correction est apparu insuffisant, montrant que le taux de croissance de la consommation de ces jours spéciaux est plus fort que celui des jours normaux. Nous avons dû introduire, pour corriger la tendance de ces jours particuliers, soit des corrections linéaires de la forme $\lambda+\mu t$, soit des corrections quadratiques de la forme $\lambda+\mu t+v t^{2}$.

Exemple: Une correction constante pour les lundi de Pâques conduit à $\lambda=0,1601$. En effectuant une correction linéaire, la correction passe de 0,1991 en 1958 à 0,1430 en 1974.

Les différents jours de fête dont nous avons dû tenir compte peuvent être répartis en trois classes :

1) les fêtes mobiles (Pâques, Ascension, Pentecôte) assez faciles à modéliser dans la mesure où elles se produisent toujours le même jour de la semaine et ont des conséquences sur un nombre limité de jours.

Exemple: Pour Pâques, nous avons introduit des corrections pour les samedis, dimanches, lundis et mardis, c'est-à-dire 5 paramètres, la correction étant linéaire pour les lundis.

2) les fêtes à date fixe ( $1^{\text {er }}$ mai, 8 mai, 14 juillet, 15 août, $1^{\text {er }}$ novembre, 11 novembre, 25 décembre, $1^{\text {er }}$ janvier) beaucoup plus difficiles à prendre en compte dans la mesure où elles ont des conséquences différentes suivant le jour de la semaine où elles se produisent, bien que le nombre de jours concernés soit faible.

Exemple: Pour la Fête du travail, nous introduisons des corrections pour le $1^{\text {er }}$ mai (constante pour les samedis et les dimanches, linéaire pour les autres jours), pour le 30 avril (constante pour les lundis), pour le 2 mai (constante pour les mardis, jeudis, vendredis, dimanches), soit 10 paramètres.

3) les vacances d'été (rappelons qu'en France, le niveau de l'activité industrielle est environ deux fois plus faible pendant le mois d'août) extrêmement difficiles à prendre en compte, dans la mesure où les conséquences dépendent du jour de la semaine où se produit le premier août, et où le nombre de jours concernés est d'environ 40 .

Le développement d'une succession de modèles ARIMA, prenant progressivement en compte un nombre croissant de perturbations, a permis de mettre au point un modèle dont les caractéristiques sur le plan statistique sont acceptables, sans être cependant parfaites.

Le dernier ajustement du modèle (2) comprend 138 paramètres. Les 3 paramètres du modèle ARIMA tenant compte des perturbations sont :

$$
\phi_{1}=0,8177 \quad \theta_{7}=0,7877 \quad \theta_{365}=0,7886
$$


au lieu des premières estimations (paragraphe 2.1):

$$
\phi_{1}=0,2919 \quad \theta_{7}=0,9686 \quad \theta_{365}=0,7068
$$

Le modèle ARIMA est donc considérablement modifié; l'écart-type du résidu devient 0,00617 (c'est-à-dire $1,4 \%$ de la valeur moyenne), au lieu de 0,0263 , soit une réduction par un facteur 4 .

\subsection{Choix d'un modèle parmi les trois candidats}

Nous avons utilisé les mêmes méthodes avec les modèles (1) et (3). L'analyse des différents coefficients correcteurs, comparés avec ceux du modèle (2), montre que :

- les résultats sont pratiquement invariants pour les fêtes mobiles et les fêtes à date fixe, donnant confirmation de la pertinence de l'approche envisagée,

- les résultats sont moins stables pour les vacances d'août, ce qui semble indiquer le besoin d'un modèle plus fin encore en ce qui concerne le mois d'août.

Sur le plan global, cependant, les meilleurs résultats ont été obtenus à l'aide du modèle (1). Les estimations des trois paramètres sont :

$$
\phi_{365}=0,0661 \quad \theta_{1}=0,0991 \quad \theta_{7}=0,8256
$$

L'écart-type du résidu est de 0,00615. La fonction d'autocorrélation du résidu présente encore quelques valeurs caractéristiques en dehors de la bande de confiance à $5 \%$ du bruit blanc normal; nous relevons une estimation $r_{7}$ de l'ordre de 0,03 pouvant traduire le besoin d'un terme $\theta_{14} B^{14}$ et une estimation $r_{364}$ de l'ordre de 0,09 qui traduit les insuffisances des corrections apportées aux mois d'août.

\subsection{Simulation de l'utilisation en temps réel du modèle A.R.I.M.A. retenu}

Nous avons simulé une prévision en temps réel, à l'aide du modèle (1), pour l'année de réalisations non prises en compte dans la phase d'estimation (du $1^{\text {er }}$ juillet 1974 au 30 juin 1975). Nous avons supposé a priori qu'il n'était pas nécessaire de réestimer les divers paramètres sur toute cette période.

Soit $T$ le nombre total des observations et soit $x_{t}$ l'observation courante. Nous définissons :

$$
\begin{array}{ll}
z_{t}=\log _{10} x_{t} & t \leqslant T \\
w_{t}=z_{t}+\text { éventuelle correction } & t \leqslant T \\
\alpha_{t}=w_{t}-w_{t-1} & t \leqslant T \\
d_{t}=\alpha_{t}-\alpha_{t-7} & t \leqslant T \\
a_{t} \text { le résidu du modèle ARIMA } & t \leqslant T
\end{array}
$$

R.A.I.R.O. Recherche opérationnelle/Operations Research 
Pour prévoir $\hat{x}_{T+1}$, nous effectuons en premier lieu une prévision $\hat{z}_{T+1}$, de la manière suivante :

$$
\begin{aligned}
& \hat{d}_{T+1}=\phi_{365} d_{T-364}-\theta_{1} a_{T}-\theta_{7} a_{T-6}+\theta_{1} \theta_{7} a_{T-7} \\
& \hat{\alpha}_{T+1}=\hat{d}_{T+1}+\alpha_{T-6} \\
& \hat{w}_{T+1}=\hat{\alpha}_{T+1}+w_{T} \\
& \hat{z}_{T+1}=\hat{w}_{T+1}-\text { éventuelle correction. }
\end{aligned}
$$

Puis, bien que cela soit une approximation [9], nous calculons simplement :

$$
\hat{x}_{T+1}=10^{\hat{z}_{T+1}}
$$

L'erreur de prévision à l'instant $T+1$ est alors :

$$
a_{T+1}=\log _{10} x_{T+1}-\hat{z}_{T+1}
$$

Avant de prévoir l'énergie du jour suivant, il convient de mettre à jour les vecteurs suivants :

$$
\begin{aligned}
z_{T+1} & =\log _{10} x_{T+1} \\
w_{T+1} & =z_{T+1}+\text { correction éventuelle } \\
\alpha_{T+1} & =w_{T+1}-w_{T} \\
d_{T+1} & =\alpha_{T+1}-\alpha_{T-6}
\end{aligned}
$$

L'écart-type de la prévision à un jour, estimé sur les 365 erreurs de prévision est de 0,00771 , ce qui correspond à un écart-type de $1,7 \%$ de la valeur moyenne, qui confirme la valeur obtenue après l'estimation (voir paragraphe 2.3). En estimant l'écart-type sur les seuls jours normaux (c'est-à-dire en excluant jours de fête et de vacances), on trouve une valeur beaucoup plus faible, de 0,00514 , soit un écart-type de $1,2 \%$ de la valeur moyenne. La figure 3 compare pour deux semaines non perturbées par les jours de fête, prévision et réalisation. Cet exemple, choisi au hasard, reflète bien la faiblesse de l'écarttype pour les jours normaux.

Les résidus sont en général à l'intérieur ou au voisinage de la bande de confiance à $5 \%$ du bruit blanc normal (d'écart-type 0,00771). Quatre d'entre eux apparaissent nettement en dehors de la bande de confiance (ils se situent entre 3 et 4 écarts-types) :

(1) $1^{\text {er }}$ août 1974 (dimanche) : un effet du mois d'août non pris en compte (coïncidence d'un dimanche et d'un $1^{\text {er }}$ août)

(2) 11 novembre 1974 (lundi) : la correction linéaire introduite semble insuffisante (ce genre de résultat nous a conduits pour Noël et le Nouvel an à introduire des corrections quadratiques) 


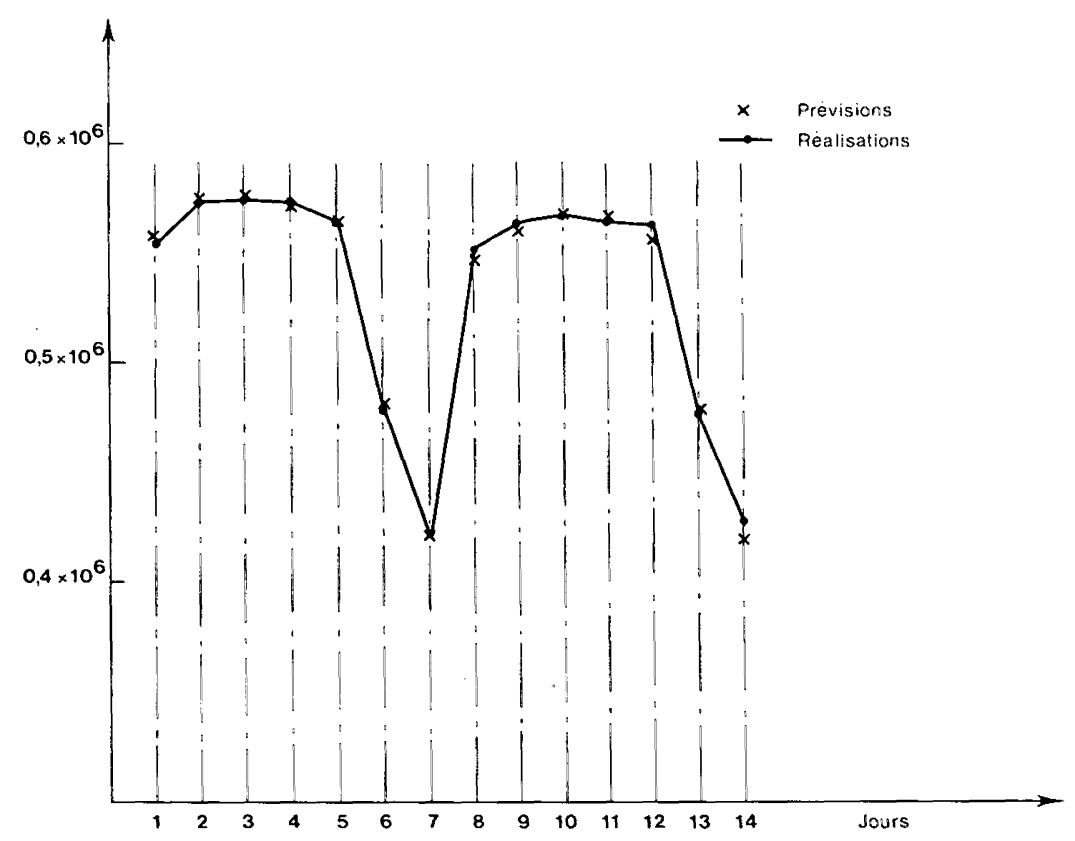

Figure 3. - Comparaison entre prévisions et réalisations pour deux semaines sans jours de fête (du 24-02-1975 au 9-03-1975).

(3) 23 décembre 1974 (lundi) : un effet des fêtes de Noël non pris en compte dans le modèle (seules les perturbations des 24, 25 et 26 Décembre ont été envisagées)

(4) 5 mai 1975 (lundi) : bien qu'il n'y ait pas de fête très voisine, cette situation demanderait une analyse approfondie permettant de savoir si cette erreur peut être considérée comme " due au hasard " ou non.

La fonction d'autocorrélation, la fonction d'autocorrélation partielle ne montrent pas d'aspect particulier (3 à $4 \%$ des estimations en dehors de la bande de confiance à $5 \%$, sauf peut-être à l'ordre 1 pour lequel les estimations semblent significatives. Le spectre ne présente pas de profil particulier.

\subsection{Quelques réflexions sur le modèle mis au point}

Ainsi que nous pouvions le pressentir a priori, la modélisation de la série des énergies journalières est loin d'être une chose aisée en raison de l'abondance des jours de fête qui ont une influence notable sur la demande d'énergie en France. 
La mise au point d'une succession de modèles ARIMA, avec prise en compte progressive d'un nombre croissant de perturbations, a permis de réaliser un modèle dont les caractéristiques sur un plan statistique sont, sans être encore parfaites, déjà convenables.

Nous obtenons un dernier modèle dont le nombre de paramètres est très important, mais dont très peu concernent le modèle ARIMA proprement dit. Ce qui peut sembler être une prolifération de coefficients correcteurs mérite ici une explication. La correction des perturbations introduites par les très nombreux jours particuliers relevait de deux soucis :

1) éliminer les perturbations pour que le modèle ARIMA s'adapte le mieux possible aux jours normaux. L'attitude extrême à cet égard aurait été d'introduire un coefficient chaque fois qu'un jour perturbé se serait présenté. Ceci aurait évidemment conduit à un nombre de paramètres extrêmement élevé;

2) essayer de comprendre les perturbations en se proposant d'en dégager des mécanismes généraux et de se donner les moyens de les prévoir.

Il fallait donc trouver un compromis entre le désir d'obtenir un modèle ARIMA le plus pur possible et celui de mettre en évidence un certain nombre de mécanismes sous-jacents aux perturbations de la série. C'est ce souci qui nous a conduits à accroître, par étapes successives, le nombre de paramètres des modèles et c'est la seule complexité des phénomènes qui en a provoqué l'importante augmentation.

Nous pensons que les résultats de l'expérience de prévision en temps réel sont tout à fait encourageants et donnent une bonne validation de l'approche que nous avons développée.

\section{CONCLUSIONS ET PERSPECTIVES}

Notre modèle de prévision à très court terme des énergies journalières produites par Electricité de France, possède déjà de bonnes propriétés. Des compléments du deuxième ordre pourraient encore être apportés pour les vacances du mois d'août et pour quelques fêtes non prises en compte (SaintEloi et Sainte-Barbe). L'actualisation de nos travaux (utilisation des dernières années de statistiques) devrait sans doute induire d'autres compléments, dont l'un se fait déjà sentir sur la figure 2 : les fêtes de Noël et du Nouvel An commencent à induire un " écrasement " prononcé de la série, analogue somme toute à celui du mois d'août (ce phénomène était peu sensible jusqu'en 1974). Un autre complément important serait de déterminer une transformation " un peu moins forte » que la transformation logarithmique utilisée.

vol. $13, \mathrm{n}^{\circ} 1$, février 1979 
Il conviendrait également de mettre au point une fonction de transfert reliant énergie et température. L'annexe III donne les premières idées dans cette direction en utilisant la série des températures moyennes journalières relevées à Paris-Montsouris (cette série représente bien, en une première approximation, l'influence de la température sur la demande d'énergie totale en France).

La technique d'optimisation retenue, utilisant le code GRGA [11], pourrait également donner lieu à une analyse spécifique. Les gros modèles auxquels nous sommes arrivés demandaient des temps de calcul importants pour leur estimation. Il faut voir d'ailleurs dans ce fait la raison pour laquelle toutes les voies de recherche complémentaires définies ci-dessus n'ont pu être explorées aussi bien que nous l'aurions souhaité.

Une extension très intéressante des travaux entrepris serait d'envisager la mise au point de modèles de prévision à très court terme de la courbe de charge. On peut déjà utiliser nos résultats et les « ventiler " à l'aide des coefficients de modulation $\pi$ (tableau 1). Il serait évidemment très intéressant d'effectuer des prévisions directement par pas horaire à l'aide d'un modèle ARIMA. Il ne faut pas se cacher que, si la modélisation des jours normaux ne doit pas poser de difficultés particulières, la prise en compte des perturbations des jours de fête s'avère a priori d'une extrême complexité.

Nous voudrions terminer par une courte remarque sur les temps de calcul mis en jeu par le développement de notre approche. Ils peuvent être très élevés au niveau de la phase d'estimation des paramètres du modèle retenu.

Au niveau de la prévision, les temps de calcul sont au contraire extrêmement faibles. La prévision à un jour est, dans le cadre du modèle retenu pour les énergies journalières, obtenue de la manière suivante :

1) calcul de $\hat{w}_{t}$ suivant la formule :

$$
\begin{aligned}
\hat{w}_{t}=w_{t-1}+w_{t-7}-w_{t-8}-0,06608 w_{t-365}-0,06608 w_{t-372} \\
+0,06608 w_{t-373}-0,09906 a_{t-1}-0,82560 a_{t-7}+0,08178 a_{t-8}
\end{aligned}
$$

c'est-à-dire quelques additions et multiplications,

2) si le jour $t$ est soumis à correction, apporter la modification convenable, c'est-à-dire quelques tests pour trouver les bons coefficients correcteurs, suivis de quelques additions et multiplications,

3) exponentiation du résultat pour obtenir la prévision effective en $M W h$.

Les temps de calcul nécessaires à une prévision sont donc extrêmement faibles (de l'ordre de quelques millièmes de seconde). De plus, en ayant adopté une série assez longue, il est probable que la réactualisation des coefficients estimés n'interviendrait que peu souvent (une fois par mois dans le pire des 
cas); en tout état de cause, il serait très facile de provoquer un déclenchement quasi automatique d'une réestimation des paramètres : il suffit par exemple de suivre les erreurs de prévision et d'en estimer diverses propriétés (le corrélogramme ou le spectre par exemple) et de déclencher la procédure d'ajustement dès qu'une hypothèse de bruit blanc, définie a priori, n'est plus satisfaite. Par ailleurs, le modèle aurait alors la même structure et on pourrait calculer exactement les dérivées utilisées par la méthode de gradient, ce qui conduirait à des temps d'estimation nettement plus faibles que ceux obtenus jusqu'alors.

\section{ANNEXE 1}

ESTIMATION DES PROPRIÉTÉS DUं PREMIER ET DU DEUXIÈME ORDRE D'UNE SÉRIE CHRONOLOGIQUE STATIONNAIRE

Soient $x_{1}, x_{2}, \ldots, x_{n}$ les diverses observations. Nous définissons: la moyenne

la fonction d'autocovariance $C($ i $)$ :

$$
\bar{x}=\frac{1}{n} \sum_{t=1}^{n} x_{t}
$$

$$
C(i)=\frac{1}{n-i+1}\left[\sum_{t=i}^{n} x_{t} x_{t-i+1}-\frac{1}{n-i+1} \sum_{t=i}^{n} x_{t} \sum_{t=1}^{n-i+1} x_{t}\right]
$$

la fonction d'autocorrélation $r(i)$ :

$$
r(i)=\frac{C(i)}{C(0)}
$$

la fonction d'autocorrélation partielle $P(i, i)$ (Méthode de Durbin [5])

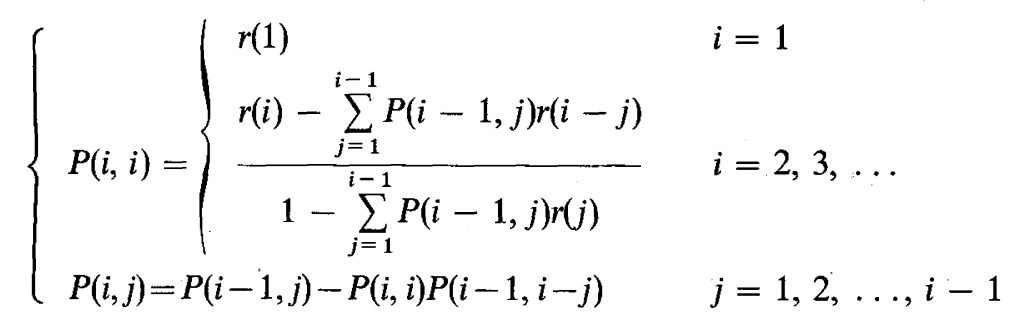

la transformée de l'autocovariance $T(i)$ (Estimation au sens de Tukey-Hanning [6])

$$
T(i)=\sum_{j=1}^{m+1} C^{\prime}(j) \cos \frac{(j-1)(i-1)}{m} \pi \quad i=1, \ldots, m+1
$$

$$
\begin{array}{lll}
\text { où : } & C^{\prime}(j)=C(j) & \text { pour } \quad j=1, m+1 \\
& C^{\prime}(j)=2 C(j) & \text { pour } \quad j=2,3, \ldots, m
\end{array}
$$

vol. $13, \mathrm{n}^{\circ} 1$, février 1979 
le spectre (Estimation au sens de Tukey-Hanning [6])

$$
\begin{aligned}
& S(1) \quad=0,5 T(1)+0,5 T(2) \\
& S(i) \quad=0,25 T(i-1)+0,5 T(i)+0,25 T(i+1) \quad i=2, \ldots, m \\
& S(m+1)=0,5 T(m)+0,5 T(m+1)
\end{aligned}
$$

\section{ANNEXE II}

\section{ESTIMATION DES MODÈLES A.R.I.M.A.}

Nous n'avons considéré que des estimations au sens des moindres carrés, l'hypothèse de normalité des résidus ayant toujours été acceptable. L'estimation des paramètres des modèles ARMA peut se définir (sur un $\operatorname{ARMA}(2,1)$ pris à titre d'exemple), comme étant la résolution du programme mathématique :

$$
\underset{\phi_{1}, \phi_{2}, \theta_{1}}{\operatorname{Min}_{t}} \sum_{t=1}^{T} a_{t}^{2} ; a_{t}=w_{t}-\phi_{1} w_{t-1}-\phi_{2} w_{t-2}+\theta_{1} a_{t-1} \quad t=1, \ldots, T
$$

plus un certain nombre de conditions assurant la stationnarité et l'inversibilité du processus, pour notre exemple :

$\phi_{2}+\phi_{1} \leqslant 2 ; \quad \phi_{2}-\phi_{1}<1 ; \quad-1 \leqslant \phi_{2} \leqslant+1 ; \quad-1 \leqslant \theta_{1} \leqslant+1$

REMARQUE : La relation de récurrence (1) pose un problème d'initialisation. Pour $t \leqslant 2$, elle fait appel à des quantités non disponibles. A cet égard, plusieurs solutions sont possibles :

1) introduire les variables non connues comme paramètres à optimiser, c'està-dire résoudre :

$$
\underset{\phi_{1}, \phi_{2}, \theta_{1}, a_{0}, x_{0}, w_{-1}}{\operatorname{Min}} \sum_{t=1}^{r} a_{t}^{2}
$$

Cette solution est envisageable pour des modèles non saisonniers. Par contre, le nombre de paramètres à ajouter devient très important pour des modèles saisonniers de période longue (environ 365 paramètres seraient nécessaires pour nos modèles de prévision de l'énergie journalière).

2) utiliser la technique de "backforecasting " développée par Box et Jenkins ([4]) en particulier lorsque la série est courte. Cette solution complique l'implantation sur ordinateur des modèles envisagés ;

3 ) introduire des valeurs arbitraires pour certaines variables ( 0 par exemple) lorsque la série est longue, et prendre un anneau de garde, c'est-à-dire résoudre un problème de la forme

$$
\operatorname{Min}_{\phi_{1}, \phi_{2}, \theta_{1}} \sum_{t=T_{1}}^{T} a_{t}^{2}
$$

R.A.I.R.O. Recherche opérationnelle/Operations Research 
C'est la solution que nous avons retenue, nos séries étant exceptionnellement longues.

L'estimation d'un modèle ARMA conduit donc en général à la résolution d'un programme non linéaire, sous des contraintes qui deviennent de plus en plus complexes à mesure que le degré du processus augmente. Heureusement, l'expérience numérique montre que si l'identification du processus est correcte, alors le résultat de l'estimation sera un processus stationnaire et inversible sans tenir compte explicitement des contraintes correspondantes.

Box et Jenkins [4] recommandent un algorithme dérivé de celui de Marquardt [12]. Nous ne l'avons pas adopté pour les deux raisons principales suivantes : d'une part, il est nécessaire de calculer les $\frac{\partial a_{t}}{\partial \beta_{i}}$ (les $\beta_{i}$ étant les différents paramètres); pour nos plus gros modèles, le volume d'informations correspondant aurait été incompatible avec un programme informatique " in core "; d'autre part, il semble nécessaire d'avoir de bonnes estimations initiales des paramètres pour obtenir une convergence acceptable. Les calculs préalables auraient été considérables pour la série des énergies.

La technique d'optimisation adoptée, utilisant le code GRGA [11] est plus générale et ne demande que le calcul des dérivées $\frac{\partial \Sigma a_{t}^{2}}{\partial \beta_{i}}$ (ce qui supprime toute difficulté de stockage en mémoire centrale). Après plusieurs essais prenant en compte les contraintes (conditions de stationnarité et d'inversibilité), le code a été utilisé comme un code d'optimisation sans contrainte (méthode des gradients conjugués [13] à la date de nos travaux).

La technique d'optimisation retenue devrait cependant donner lieu à une analyse spécifique. GRGA permettait d'aborder sans difficulté l'estimation des divers modèles tant que le nombre de paramètres n'était pas trop important (avec $6000 a_{t}, 4$ minutes avec 19 paramètres, 30 minutes avec 40 paramètres sur un I.B.M. 370-168). Par contre, un nombre important de paramètres nous conduisait à des temps de calcul très élevés.

Les temps de calcul observés proviennent pour une bonne part du mode de calcul des dérivées, par différences centrales, demandant pour 100 variables, 200 appels de fonction pour calculer le gradient, soient 200 explorations de la série temporelle : une formule exacte pour le gradient ne demanderait qu'une seule exploration. En contrepartie, la détermination analytique du gradient exige une formulation extrêmement compliquée qui est à remettre entièrement en cause dès qu'une modification est introduite dans le modèle. Une fois le modèle fixé (pour la recherche de la meilleure transformation par exemple), il est certainement rentable d'effectuer les calculs littéraux nécessaires à l'obtention des formules; une réduction importante du temps de calcul peut en être attendue. vol. $13, \mathrm{n}^{\circ} 1$, février 1979 
Une analyse plus spécifique pourrait porter sur la technique d'optimisation proprement dite. Quelques essais utilisant un autre code [14] ont été très encourageants pour un faible nombre de paramètres : temps divisé par 2 pour 19 paramètres. Par contre, un essai sur un plus grand modèle (100 paramètres) a donné de moins bons résultats que GRGA, au bout de 100 itérations. Une poursuite des travaux dans cette direction devrait conduire à des réductions des temps d'estimation.

Nous n'avons pas, en général, estimé les écarts-types des coefficients. Il $\mathrm{y}$ a trois raisons à cela :

1) nous avons plutôt jugé le bien fondé d'un coefficient en fonction de l'analyse des diverses propriétés du second ordre (autocorrélation, autocorrélation partielle et spectre) estimées sur le résidu d'un modèle contenant ou ne contenant pas le coefficient;

2) pour les gros modèles, cette estimation aurait été lourde. Rappelons qu'il est nécessaire de calculer la matrice des dérivées secondes du critère $\Sigma a_{t}^{2}$ (ceci est très facile au niveau du principe avec le code GRGA, par une méthode de différences centrales), puis d'inverser cette matrice, puis de la multiplier par deux fois la variance du résidu. La diagonale donne les variances des coefficients et les autres éléments les covariances entre les coefficients;

3) de toute façon, nous avons ajusté nos modèles avec un nombre important d'observations, et l'écart-type du résidu est faible, ce qui conduit à des écartstypes des coefficients faibles (de $10^{-4}$ à $10^{-3}$ comme nous l'ont indiqué quelques estimations faites sur des modèles simples mis au point dans le cadre de cette étude).

\section{ANNEXE III}

PREMIER ESSAI D'IDENTIFICATION D'UNE FONCTION DE TRANSFERT LIANT L'ÉNERGIE JOURNALIÊRE A LA TEMPÉRATURE JOURNALIẼRE

Différentes analyses effectuées au sein d'E. D. F. ont montré que la température moyenne journalière relevée à Paris-Montsouris représente, en une première approximation, l'influence de la température sur la demande d'énergie électrique en France. C'est donc la série que nous avons utilisée pour cette analyse.

Soient $x_{t}, y_{t}$ les observations courantes de la température et de l'énergie et soit $n$ le nombre total d'observations. Transformons en premier lieu les deux séries pour induire leur stationnarité simultanée, c'est-à-dire calculons :

$$
\begin{array}{rlrl}
\mu_{t} & =\nabla \nabla_{7} \nabla_{365} x_{t} & t & =1, \ldots, \\
v_{t} & =\log _{10} y_{t}+\text { correction pour les jours de fête } & t & =1, \ldots, \\
w_{t} & =\nabla \nabla_{7} \nabla_{365} v_{t} & t & =1, \ldots
\end{array}
$$

R.A.I.R.O. Recherche opérationnelle/Operations Research 
Nous calculons la covariance des deux séries ainsi transformées :

$$
C C(i)=\frac{1}{n-i+1}\left[\sum_{t=i}^{n} w_{t} \mu_{t-i+1}-\frac{1}{n-i+1} \sum_{t=i}^{n} w_{t} \sum_{t=1}^{n-i+1} \mu_{t}\right]
$$

Ceci conduit à la fonction de corrélation entre les deux séries. L'analyse de cette estimation montre :

1) une forte valeur à l'ordre 0 , suivi de valeurs plus faibles aux ordres 1 et 2 , le tout semblant décroître de manière exponentielle;

2) une forte valeur à l'ordre 7 et rien de significatif à l'ordre 14 (avec un satellite à l'ordre 8, certainement induit par les valeurs des ordres 1 et 7);

3) une forte valeur à l'ordre 365 et rien de significatif à l'ordre 730 (avec un satellite à l'ordre 366, certainement induit par les valeurs des ordres 1 et 365).

Ceci conduit à postuler la fonction de transfert suivante :

$$
\left(1-\delta_{1} B\right) v_{t}=\omega_{0}\left(1-\omega_{7} B^{7}\right)\left(1-\omega_{365} B^{365}\right) x_{t}+N_{t}
$$

où $N_{t}$ représente la « part » des $v_{t}$ qui ne peut être expliquée par la température ; pour $N_{t}$ nous adopterons bien entendu la structure du modèle endogène de la série des énergies, c'est-à-dire :

$$
\left(1-\phi_{365} B^{365}\right) \nabla \nabla_{7} N_{t}=\left(1-\theta_{1} B\right)\left(1-\theta_{7} B^{7}\right) a_{t}
$$

La combinaison de ces deux termes conduit au modèle cherché.

L'estimation du modèle conduirait à résoudre

$$
\underset{\delta_{1}, \omega_{0}, \omega_{7}, \omega_{36} 65, \theta_{1}, \theta_{7}, \phi_{365}}{\operatorname{MIN}} \sum_{t} a_{t}^{2}
$$

La technique utilisée pour les modèles endogènes est ici directement applicable.

ReMARQues: 1) Ce modèle est à considérer comme une première tentative de prise en compte de l'influence de la température sur la consommation. Les résultats de l'estimation, en particulier l'analyse des résidus, peut induire des modifications de cette première identification. Cependant, ce modèle comprend des mécanismes bien connus par ailleurs, essentiellement la variation de la sensibilité de la consommation à la température en fonction du jour dans la semaine et du jour dans l'année (termes $1-\omega_{7} B^{7}$ et $1-\omega_{365} B^{365}$ respectivement). Le terme $\left(1-\delta_{1} B\right)$ caractérise un phénomène non pris en compte dans les procédures actuelles, à savoir une certaine inertie de la consommation face aux dernières températures réalisées.

2) Pour une utilisation dans des conditions réelles de prévision, la température $x_{t}$ n'est évidemment pas connue et il conviendrait de la templacer par une prévision $\hat{x}_{t}$ (dans les méthodes actuelles, $\hat{x}_{t}=x_{t-1}$, modèle simple qui a cependant, dans la classe des modèles endogènes, des propriétés satisfaisantes).

vol. 13, n 1 , février 1979 
3) On peut aussi prendre les prévisions de la météorologie nationale, qui consistent en un couple de températures, minimale et maximale. Leur moyenne est, selon ce que semblent montrer des études en cours, un indicateur meilleur que le précédent, en ce sens que cette prévision est plus proche de sa réalisation que $x_{t-1}$ ne l'est de $x_{t}$, et que sa valeur explicative est au moins égale.

\section{ANNEXE IV}

Notations : pour une série temporelle quelconque $\left(u_{t}\right)$ :

$$
B^{k} u_{t}=u_{t-k}, \nabla_{k} u_{t}=u_{t}-u_{t-k}, \quad \nabla_{h} \nabla_{k} u_{t}=\nabla_{h}\left(\nabla_{k} u_{t}\right)
$$

\section{BIBLIOGRAPHIE}

1. J. Abadie et F. Meslier, Présentation synthétique de modèles de prévision à très court terme de l'énergie journalière produite par Électricité de France et de la température moyenne journalière relevée à Paris Montsouris, Cahier du LAMSADE, $\mathrm{n}^{\circ} 10$, octobre 1977.

2. F. MEsLIER, Contribution à l'analyse des séries chronologiques et application à la mise au point de modèles de prévision à court terme relatifs à la demande journalière d'énergie électrique en France et à la température relevée à Paris Montsouris. Thèse Université Paris IX-Dauphine, décembre 1976.

3. A. Ferrandiz, M. Penel and Y. Pioger, Short, medium and long term load forecasting models. IEEE PES Summer Meeting, Paper no A 75 430-9, July 1975.

4. G. E. P. Box and G. M. JENKINS, Time Series Analysis forecasting and control. Holden Day, San Francisco, 1970.

5. J. Durbin, The fitting of time series models. Rev. Int. Inst., 28, 1960, p. 233-244.

6. C. W. J. Granger and M. Hatanaka, Spectral analysis of economic time series. Princeton University Press, 1964.

7. G. E. P. Box and D. R. Cox, An analysis of transformations. Journ. Royal Stat. Soc., B26, 1974, p. 211-252.

8. G. E. P. Box and G. M. Jenkins, Some comments on a paper by Chatfield and Prothero and on a review by Kendall (with a reply). Journ. Royal Stat. Soc., A136, 1973, p. 337-352.

9. C. W. J. Granger and P. Newbold, Forecasting Transformed Series. Journ. Royal Stat. Soc., $\mathrm{n}^{\circ} 2,1976$, p. 189-203.

10. J. ABADIE and J. CARPENTIER, Generalization of the Wolfe reduced gradient method to the case of non linear constraint, in: Optimization, Fletcher ed., Academic Press, 1969, p. 37-47.

11. J. Abadie, Méthode du gradient réduit généralisé, le code GRGA, Note E.D.F., HI 1758, février 1975.

12. D. W. MARQUARDT, An algorithm for least squares estimation of non linear parameters. Journ. Soc. Ind. Appl. Math., 11, 1963, p. 431-441.

13. R. FletCHER and C. M. ReEVES, Function minimization by conjugate gradients. Comput. Journ., 6, 1964, p. 149-154.

14. A. A. H. HAGGAG, Étude d'algorithme d'optimisation non linéaire (une variante $d u$ GRG). Thèse Université Paris VI, novembre 1976. 